\title{
Lukrativer Staatsdienst? Lohndifferenzen zwischen öffentlichem Dienst und Privatwirtschaft
}

\author{
Markus Tepe \\ Daniela Kroos
}

Lange Zeit galt der öffentliche Dienst als Gewährleister vorbildlicher Beschäftigungsverhältnisse. Auch die Vergütungsstrategien der öffentlichen Arbeitgeber wiesen eine soziale Komponente auf und reduzierten die Kluft zwischen Besserverdienenden und denjenigen am unteren Ende der Lohnskala. Veränderte Personal- und Tarifpolitiken sowie die „Flexibilisierung“von Beschäftigungsverhältnissen werfen jedoch die Frage auf, inwieweit die bisherigen Lohndifferenzen zwischen öffentlichem Dienst und Privatwirtschaft noch bestehen. Eine Bestandsaufnahme dieser Unterschiede auf Basis der Daten des Sozio-oekonomischen Panels (SOEP) zeigt auf, welchen Einfluss Charakteristika der Beschäftigten und der Beschäftigungsverhältnisse auf die Lohndifferenz haben. ${ }^{1}$

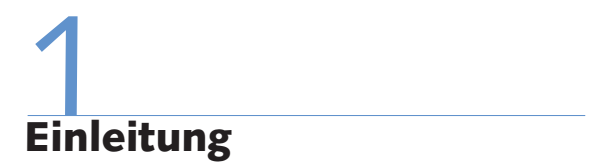

Trotz anhaltenden Stellenabbaus im öffentlichen Dienst ist der Staat mit mehr als 4,5 Mio. Beschäftigten nach wie vor der wichtigste Arbeitgeber in Deutschland (Statistisches Bundesamt 2009). In ihrer Rolle als Arbeitgeber übernehmen Bund, Länder und Kommunen nicht nur die finanzielle, sondern auch die sozialpolitische Verantwortung für einen erheblichen Teil der Erwerbsbevölkerung. In Deutschland hat sich, ebenso wie in anderen europäischen Staaten, ein öffentliches Beschäftigungsregime etabliert (Tepe et al. 2008), das durch die Selbstverpflichtung, ein ,guter Arbeitgeber" zu sein, geprägt ist. Diese Vorbildfunktion äußerte sich in den vergangenen Jahrzehnten nicht nur in gesetzlichen Regelungen, wie beispielsweise der frühzeitigen Etablierung des Gleichbehandlungsgrundsatzes oder der gezielten Integration benachteiligter Gruppen in den Arbeitsmarkt, sondern auch in den öffentlichen Tarifstrukturen. Anders als die privatwirtschaftliche Lohnpolitik strebte die staatliche Lohnpolitik einen gesellschaftlichen Ausgleich an und hielt die Kluft zwischen den hohen und den niedrigen Einkommensgruppen wesentlich geringer als dies in der Privatwirtschaft der Fall war. Mehrere Studien konnten zeigen, dass sich die tarifpolitische Vorbildrolle des öffent- lichen Dienstes zumindest bis zur Jahrtausendwende auch tatsächlich im Geldbeutel der Beschäftigten auswirkte: Während Beschäftigte in höheren Einkommensgruppen oder mit hohen Qualifikationen im öffentlichen Dienst schlechtere Verdienstchancen hatten als in der Privatwirtschaft, war bei den Beschäftigten mit geringeren Qualifikationen und in niedrigen bzw. mittleren Einkommensgruppen das Gegenteil der Fall - sie konnten im öffentlichen Dienst wesentlich mehr verdienen (Disney/ Gosling 1998; Allington/Morgan 2003; Melly 2005).

Einige der gegenwärtigen Entwicklungstendenzen der Beschäftigungsverhältnisse im öffentlichen Dienst werfen jedoch die Frage auf, ob die Lohndifferenzen zur Privatwirtschaft nach wie vor diesem Muster folgen. Der wachsende Niedriglohnbereich im öffentlichen Dienst, die Einrichtung von flexibleren Beschäftigungsverhältnissen oder der Abbau von Stellen in einfachen Tätigkeitsbereichen ${ }^{2}$ (vgl. zu diesen Punkten Czerwick 2007) sind mögliche Hinweise auf eine Erosion der bisherigen Lohnunterschiede zwischen öffentlichem Dienst und Privatwirtschaft.

Dieser Beitrag untersucht auf Basis von Querschnittsdaten des Sozio-oekonomischen Panels aus den Jahren 1995 und 2007, ob und in welcher Hinsicht vormalige Differenzen zwischen den Lohnstrukturen bei Beschäftigten öffentlicher und privater Arbeitgeber noch bestehen. Die lohnpolitische Vorbildfunktion des öffentlichen
Dienstes bezog sich jedoch nicht nur auf den Ausgleich zwischen Mitarbeiterinnen und Mitarbeitern in höheren und in niedrigeren Einkommensgruppen, sondern auch auf den Ausgleich zwischen Beschäftigtengruppen mit unterschiedlichen Arbeitsmarktchancen. Daher überprüfen wir

\footnotetext{
Der vorliegende Beitrag folgt einem Call for Papers der WSI-Mitteilungen zum Thema "Der Staat als Arbeitgeber ${ }^{\prime}$, der unter www.boeckler.de/pdf/ wsimit_2009_call_for_paper_ag_staat.pdf abgerufen werden kann. Die Autoren bedanken sich bei zwei anonymen Gutachtern für wesentliche Hinweise und Kommentare zu einer früheren Version. 2 So kam es zu einer Ausdünnung der unteren zugunsten der oberen Laufbahngruppen sowie zu einer Verschiebung des Beschäftigungsschwerpunktes von Tarifbeschäftigten zu Beamtinnen und Beamten.
}

Markus Tepe, Dr., ist Politikwissenschaftler am Zentrum für Methoden der Sozialwissen schaften, Universität Oldenburg und im Sonderforschungsbereich 597 "Staatlichkeit im Wandel ", Universität Bremen. Arbeitsschwerpunkte: Empirische Wirtschafts- und Sozialforschung, Politische Ökonomie. e-mail: Markus.Tepe@uni-oldenburg.de Daniela Kroos, Dr., ist Soziologin an der Universität Bremen, Sonderforschungsbereich 597 "Staatlichkeit im Wandel".

Arbeitsschwerpunkte: Arbeitsmarktforschung und Wohlfahrtsstaatsforschung im europäischen Vergleich.

e-mail: Daniela.Kroos@sfb597.uni-bremen.de 
zudem, wie sich der Einfluss bestimmter Charakteristika der Beschäftigten und der Beschäftigungsverhältnisse auf individuelle Bruttostundenlöhne im öffentlichen Dienst und in der Privatwirtschaft zwischen 1995 und 2007 ausgewirkt hat.

\section{2 \\ Wandel der Beschäftigungs- verhältnisse im öffentlichen Dienst}

In den vergangenen zehn Jahren hat sich das Leitbild der öffentlichen Beschäftigung tief greifend gewandelt. Während die Prinzipien des New Public Management beim europäischen Vorreiter Großbritannien bereits im Verlauf der 1980er Jahre zu einem radikalen $\mathrm{Ab}$ - und Umbau staatlicher Beschäftigung führten (Pollitt/Bouckaert 2004), gewann diese Neuorientierung des öffentlichen Dienstes erst in den 1990er Jahren in Deutschland an Einfluss (Bull 2006; Czerwick 2007). Im Rahmen der Verwaltungsmodernisierung wurde die Ausrichtung des öffentlichen Personalwesens an den Effizienzkriterien der privatwirtschaftlichen Personalführung zu einem zentralen Ziel. Obgleich Umfang und Erfolg der Personalreformen unterschiedlich bewertet werden (Reichard 2005), besteht kein Zweifel, dass sich der Charakter öffentlicher Beschäftigung mit der Einführung privatwirtschaftlicher Instrumente des Personalmanagements gewandelt hat. Durch die Gleichzeitigkeit von Stellenabbau und neuen Anforderungen an die Beschäftigten haben sich die Arbeitsbedingungen zahlreicher Beschäftigter im öffentlichen Dienst wesentlich verändert.

In vielen Bereichen des öffentlichen Dienstes wird von einer Verschlechterung der Arbeitsbedingungen und einer stark gestiegenen Arbeitsintensität berichtet, die zumindest teilweise auf Personalabbau und eine Unterfinanzierung öffentlicher Dienstleistungen zurückzuführen sind (Ahlers 2004). Darüber hinaus haben sich auch die äußeren Bedingungen der Beschäftigung im öffentlichen Dienst zumindest für Neueinsteiger zugespitzt. Selbst wenn man die aus Ausbildungsgründen befristeten Arbeitsverträge außer Acht lässt, ist die Befristungsquote im öffentlichen Dienst deutlich höher als in der Privatwirtschaft. ${ }^{3}$ Gleichzeitig hat jedoch auch ein Abbau der Beschäftigungsverhältnisse im einfachen

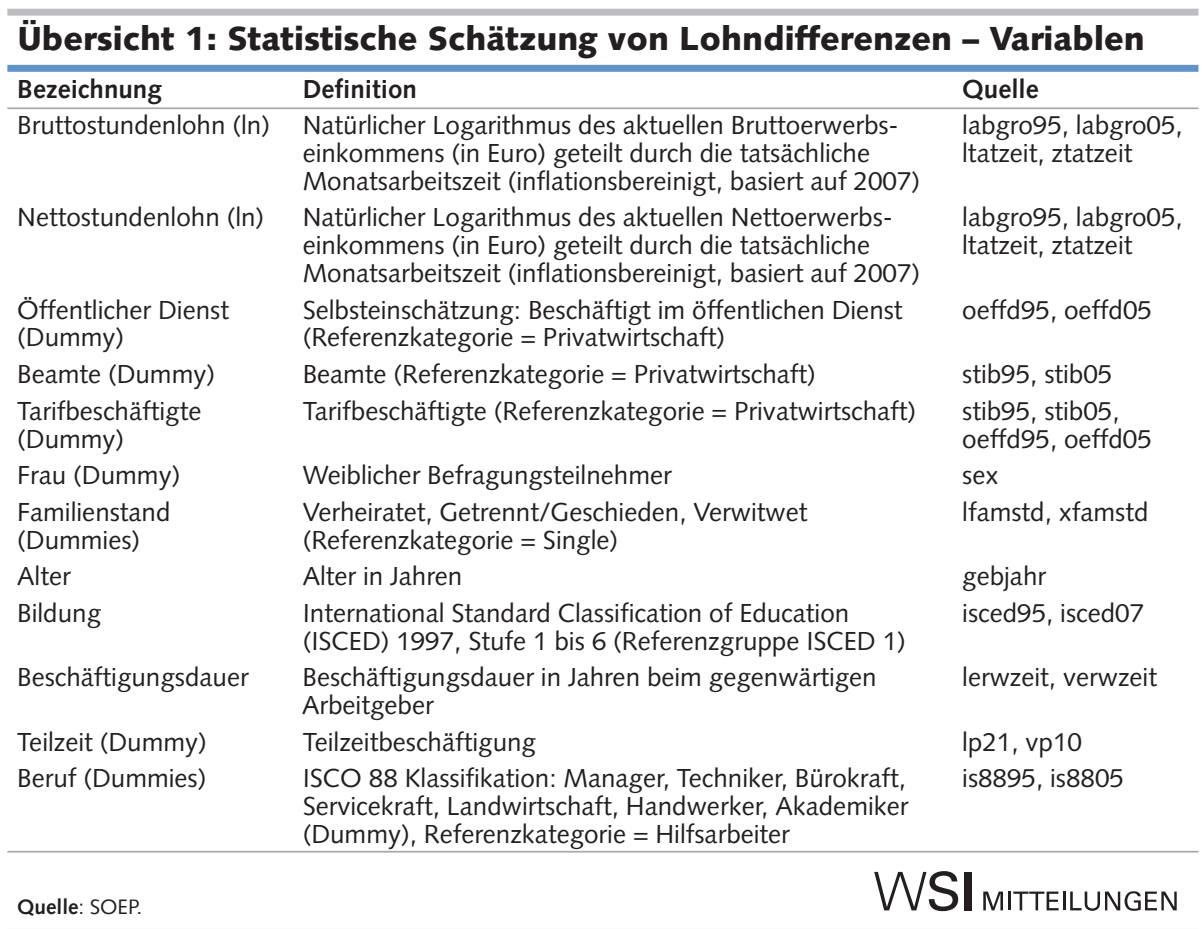

Dienst stattgefunden, der mit dem Outsourcing ganzer Beschäftigungssparten wie der Reinigungsdienste verbunden ist, in denen die Privatwirtschaft deutlich geringere Löhne zahlt (vgl. für einen Überblick Czerwick 2007; zur Rolle von Outsourcing in Krankenhäusern Jaehrling 2007). Durch den Tarifvertrag für den öffentlichen Dienst (TVÖD) und den Tarifvertrag für den öffentlichen Dienst der Länder (TV-L) hat sich aber auch im öffentlichen Dienst der Anteil niedrig entlohnter Beschäftigter erhöht. Daher stellt sich die Frage, ob der schon lange bestehende Finanzdruck der öffentlichen Arbeitgeber, der über die Verringerung der Personalkosten ein potenzielles Ventil findet, zu veränderten Einkommensstrukturen im öffentlichen Dienst beigetragen hat.

Wir untersuchen auf Basis der Daten des Sozio-oekonomischen Panels, wie sich die realen, individuellen Bruttostundenlöhne von voll- und teilzeitbeschäftigten Personen im Alter zwischen 18 und 64 Jahren zwischen 1995 und 2007 verändert haben. ${ }^{4}$ Auszubildende, die in der Regel unterdurchschnittliche Vergütungen beziehen, wurden aus dem Sample ausgeschlossen. Die Auswahl der Untersuchungszeitpunkte erfolgte in der doppelten Absicht, ein aktuelles Bild der Lohndifferenzen zu zeichnen und die Entwicklung für Gesamtdeutschland abzubilden. Wir nehmen an, dass sich der öffentliche Dienst in Ostdeutschland in den unmittelbaren Jahren nach der Wiedervereinigung in einer Übergangs- und Konsolidierungsphase befand, sodass wir erst das Jahr 1995 als geeigneten Vergleichszeitpunkt gewählt haben.

Um den Einfluss weiterer individueller Merkmale auf das Erwerbseinkommen konstant zu halten, beinhaltet das statistische Modell eine Reihe sozio-ökonomischer Kontrollvariablen; dazu zählen Beamtenstatus, Familienstand, Beschäftigungsdauer, Beruf und Teilzeitbeschäftigung (Übersicht 1; Tabelle 1). Aufgrund der deutlichen Unterschiede in den geschlechtsspezifischen Prozessen der Lohndetermination wird die Analyse generell für Männer und Frauen getrennt durchgeführt. Den Effekt öffentlicher Beschäftigung auf den logarithmierten Bruttostundenlohn ermitteln wir mithilfe einer Quantilsregression, die es uns ermöglicht, Lohndifferenzen zwischen öffentlichem Dienst und Privatwirtschaft nach Einkommensgruppen zu unterscheiden (Koenker/Bassett 1978; Hao/Naiman 2007). Die Quantilsregression schätzt, im Gegensatz zur Kleinst-Quadrat (KQ)-

3 Die Befristungsquote in der Privatwirtschaft betrug ohne ausbildungsbedingt befristete Arbeitsverträge sowie Wehr- und Zivildienstleistende im Jahr $20057,4 \%$, im öffentlichen Dienst dagegen 13,1 \% (Mikrozensus 2005, eigene Berechnung).

4 Der Bruttostundenlohn ist definiert als das Verhältnis aus dem aktuellen Bruttoerwerbseinkommen (in Euro) und der tatsächlichen Monatsarbeitszeit. Die Inflationsbereinigung basiert auf dem Jahr 2007. 
Tabelle 1: Statistische Schätzung von Lohndifferenzen Deskriptive Statistik (Mittelwerte für 2007)

\begin{tabular}{lrrrr} 
& Privat & Öffentlich & Privat & Öffentlich \\
& \multicolumn{2}{c}{ Männer } & \multicolumn{2}{c}{ Frauen } \\
\hline Bruttostundenlohn (In) & 4,14 & 4,28 & 3,84 & 4,13 \\
Nettostundenlohn (In) & 3,71 & 3,92 & 3,40 & 3,69 \\
verheiratet (Dummy) & 0,64 & 0,69 & 0,58 & 0,63 \\
getrennt/geschieden (Dummy) & 0,10 & 0,12 & 0,15 & 0,14 \\
verwitwet (Dummy) & 0,00 & 0,00 & 0,02 & 0,02 \\
Alter & 42,95 & 46,13 & 42,02 & 44,61 \\
International Standard Classification & 3,97 & 4,41 & 3,79 & 4,46 \\
of Education (ISCED) (1-6) & & & & 14,04 \\
Beschäftigungsdauer (Jahre) & 11,26 & 16,95 & 9,14 & 0,45 \\
Teilzeitbeschäftigung & & & 0,43 & 0,02 \\
(Dummy) & 0,03 & 0,05 & 0,05 & 0,40 \\
Manager (Dummy) & 0,11 & 0,05 & 0,28 & 0,12 \\
Techniker (Dummy) & 0,17 & 0,20 & 0,20 & 0,06 \\
Büroangestellter (Dummy) & 0,07 & 0,06 & 0,19 & 0,00 \\
Servicekraft (Dummy) & 0,03 & 0,07 & 0,01 & 0,01 \\
Landwirtschaft (Dummy) & 0,02 & 0,01 & 0,04 & 0,35 \\
Handwerker (Dummy) & 0,27 & 0,06 & 0,12 & \\
Akademiker (Dummy) & 0,17 & 0,41 & VSI MITTEILUNGEN
\end{tabular}

\section{Abb. 1: Lohndifferenz zwischen öffentlichem Dienst und \\ Privatwirtschaft, West-Ost-Vergleich 1995 und 2007 - in \% -}
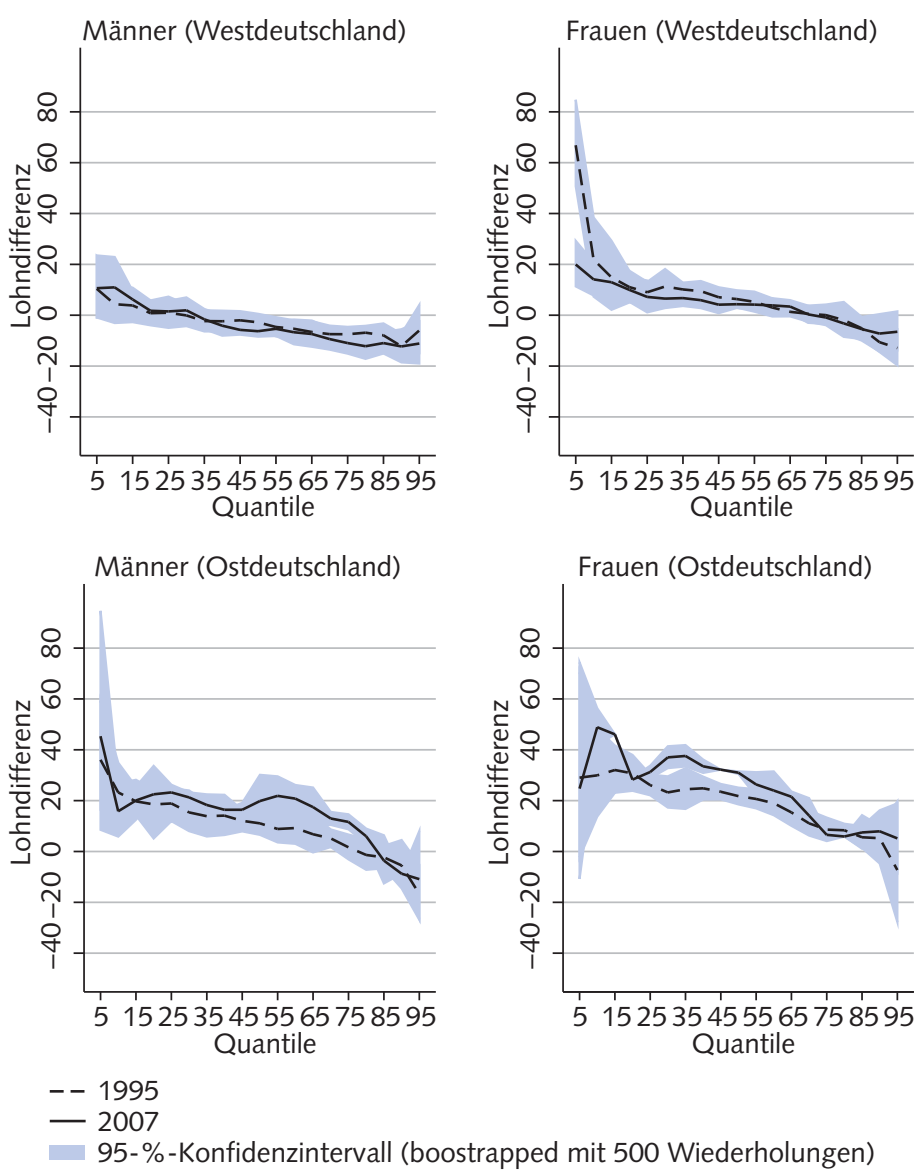

Methode: Die in den vier Teilgrafiken dargestellten Schätzergebnisse basieren auf acht separaten Samples (jeweils 18-64-Jährige in Voll- oder Teilzeitbeschäftigung). Abhängige Variable: Bruttostundenlohn(In). Kontrollvariablen: Beamter, Familienstand, Alter, Bildung, Beschäftigungsdauer, Teilzeit, Beruf (Querschnittgewichte verwendet). Quelle: SOEP; Berechnungen der Autoren.
Regression, keine bedingte MittelwertFunktion, sondern eine bedingte MedianFunktion auf Basis der Minimierung der Summe der absoluten Fehler. Auf diese Weise wird der Effekt der unabhängigen Variablen auf den Bruttostundenlohn an unterschiedlichen Abschnitten der Einkommensverteilung getestet. Das macht die Quantilsregression im Vergleich zur KQRegression robust gegen Extremwerte in der abhängigen Variablen (Hao/Naiman 2007). Wir schätzen die prozentuale Lohndifferenz für 19 Quantile $(0.05,0.10, \ldots$, 0.95) und verwenden die SOEP-Querschnittsgewichte für die Wellen 1997 und 2007.5

\section{Lohndifferenzen bei unterschiedlichen Beschäftigtengruppen}

Die Lohndifferenzen zwischen Beschäftigten in der Privatwirtschaft und dem öffentlichen Dienst unterscheiden sich nicht nur hinsichtlich ihrer Position in der Einkommensverteilung. Auch regionale Unterschiede, persönliche Merkmale der Beschäftigten und die Form des Beschäftigungsverhältnisses beeinflussen die Lukrativität der Tätigkeit im öffentlichen Dienst. Im Folgenden wollen wir die Lohndifferenzen zwischen öffentlichem Dienst und Privatwirtschaft in Abhängigkeit von diesen Faktoren beurteilen. Die Abbildungen 1, 2 und 3 zeigen die prozentuale Lohndifferenz zwischen öffentlicher und privater Beschäftigung (y-Achse) für unsere 19 Einkommensperzentile (x-Achse). Die gestrichelte Linie bezieht sich auf das Jahr 1995, die durchgezogene Linie auf das Jahr 2007;

\footnotetext{
5 Die statistischen Modelle wurden mit dem qreg Kommando in Stata 10 geschätzt (Koenker/Bassett 1978). Mit diesem Verfahren ist es leider nicht möglich, Standardfehler und das 95-\%-Konfidenzintervall des Koeffizienten via boostrapping zu ermitteln. Dieser Schwachpunkt kann mit der Simultaneous Quantile Regression (sqreg) behoben werden. Die Wahl zwischen Quantilsregression nach Koenker/ Bassett (1978) und Simultaneous Quantile Regression hat keinen Einfluss auf den geschätzten Koeffizienten. Die in Stata 10 implementierte Simultaneous Quantile Regression erlaubt jedoch nicht die Verwendung von Gewichten. Die Ergebnisse der ungewichteten Simultaneous Quantil Regression mit boostrapped Standardfehlern (500 Wiederholungen), der Datensatz und die Syntax-Datei werden bei Interesse zur Verfügung gestellt.
} 
die graue Fläche zwischen den Graphen markiert das 95-\%-Konfidenzintervall. ${ }^{6}$ Angaben zur Spezifikation des statistischen Modells und dem zugrunde liegenden Sample sind unterhalb der Abbildungen aufgeführt.

\subsection{OST-WEST-VERGLEICH}

Aufgrund unterschiedlicher Lebenshaltungskosten erhalten Beamtinnen und Beamte sowie Tarifbeschäftigte in den ostdeutschen Bundesländern geringere Bruttostundenlöhne als Beschäftigte in den westdeutschen Bundesländern. Im TVÖD wurde die Unterscheidung zwischen ostund westdeutschen Beschäftigten des Bundes erst 2008 aufgehoben. Der zuvor geltende Bundesangestelltentarif (BAT) sah ebenfalls eine Unterscheidung zwischen Ost- und Westdeutschland vor. Bei den Tarifbeschäftigten der Kommunen (TVÖD), der Länder (TV-L) sowie bei den Beamtinnen und Beamten gilt dies nach wie vor. Wie sich die Einkommenssituation der öffentlich Beschäftigten jedoch zu den ebenfalls niedrigeren Arbeitsentgelten in der ostdeutschen Privatwirtschaft verhält, ist eine bislang ungeklärte Frage. Dem öffentlichen Dienst kommt die schwierige Aufgabe zu, die Balance zwischen den aufgrund niedrigerer Lebenshaltungskosten geringeren ostdeutschen Tarifen und einer zu starken Annäherung an die teilweise extrem niedrigen Löhne der ostdeutschen Privatwirtschaft zu halten. Bereits heute wird eine zunehmende Konkurrenz der Bundesländer bei der Rekrutierung von Beschäftigten beobachtet (Seidel et al. 2008). So haben Berlin und Hessen die Tarifgemeinschaft deutscher Länder verlassen, um ihren Vergütungsspielraum bei den Tarifbeschäftigten zu vergrößern. Auch die in der Föderalismusreform vereinbarte Übertragung der Gesetzgebungskompetenz zur Besamtenbesoldung auf die Länder wird vermutlich dazu führen, dass die Bundesländer in stärkerem Ausmaß um die besten Beamten kämpfen müssen.

Abbildung 1 zeigt, wie sich die prozentuale Lohndifferenz zwischen öffentlichem Dienst und Privatwirtschaft für Männer und Frauen zwischen Ost- und Westdeutschland unterscheidet. Mit steigendem Einkommen verschlechtert sich demnach der Bruttostundenlohn der Beschäftigten im öffentlichen Dienst relativ zur Privatwirtschaft. Bei in Westdeutschland beschäftigten Männern hat sich die positive
Lohndifferenz zur Privatwirtschaft im unteren Einkommensbereich zwischen 1995 und 2007 ebenso vergrößert wie die negative Einkommensdifferenz im hohen Einkommensbereich. Da sich die 95-\%-Konfidenzintervalle jedoch deutlich überlagern, kann kein systematischer Unterschied zwischen der Lohndifferenz 1995 und 2007 beobachtet werden. In Bezug auf in Ostdeutschland beschäftigte Männer zeigt sich eine andere Entwicklung. Öffentlich beschäftigte Männer in den ostdeutschen Bundesländern hatten sowohl 1995 als auch 2007 in den unteren und mittleren Einkommensgruppen höhere Bruttostundenlöhne als privatwirtschaftlich Beschäftigte; die positive Lohndifferenz zur Privatwirtschaft reichte zudem zu beiden Untersuchungszeitpunkten bis in das achte Einkommensdezil hinein.

Die Lohndifferenzen zwischen in Westdeutschland öffentlich oder privatwirtschaftlich beschäftigten Frauen unterscheiden sich deutlich von denen der Männer. Zunächst ist die positive Lohndifferenz zur Privatwirtschaft größer als bei den Männern und schließt auch noch Frauen in mittleren Einkommensbereichen ein. Erst ab dem 75. Einkommensperzentil wird die Lohndifferenz zur Privatwirtschaft negativ. Bei den in Ostdeutschland beschäftigten Frauen ist die Lohndifferenz zur Privatwirtschaft bis in die höchsten Einkommensbereiche positiv und größer als bei den Männern. Zudem hat sich die positive Lohndifferenz bei den Frauen zumindest bis in die mittleren Einkommensbereiche hinein zwischen 1995 und 2007 tendenziell vergrößert.

Die Gleichbehandlung von Männern und Frauen ist ein zentrales Anliegen des Staates als ,guter Arbeitgeber“. Frauen sollen nicht nur beim Eintritt in den öffentlichen Dienst gleichgestellt sein; auch die Regelungen der Tarifverträge und des Beamtenrechts tragen dazu bei, Aufstiegswege für Frauen zu erleichtern. Bisherige Arbeiten konnten zeigen, dass sich dieser Anspruch staatlicher Lohnpolitik auch in der geschlechtsspezifischen Lohndifferenz zwischen öffentlicher und privater Beschäftigung widerspiegelt (Dustmann/Van Soest 1997; Dustmann/Van Soest 1998; Melly 2005; Ponthieux/Meurs 2005). Das Erwerbseinkommen von Frauen im öffentlichen Dienst ist in der Regel höher als das Erwerbseinkommen von Frauen in der Privatwirtschaft. Unsere Ergebnisse können als Beleg dafür gelten, dass das Lohngefüge des öffentlichen Dienstes zumindest im Vergleich mit der Privatwirtschaft nach wie vor geschlechterpolitisch vorbildlich ist. Die berufliche Geschlechtersegregation im öffentlichen Dienst, die unsere Daten nicht abbilden, könnte dieses Bild allerdings relativieren. Der Anteil weiblicher Beschäftigter ist im öffentlichen Dienst nicht zuletzt deshalb wesentlich höher als in der Privatwirtschaft, weil im öffentlichen Dienst insbesondere im Gesundheits- oder Erziehungswesen eine Reihe von Branchen und Berufsbildern dominiert, die als weibliche Arbeitsfelder gelten.

Ein neues Licht werfen unsere Ergebnisse auf die Attraktivität des öffentlichen Dienstes in den neuen Bundesländern. Der öffentliche Dienst ist dem allgemeinen Abwärtstrend im Lohngefüge der ostdeutschen Privatwirtschaft offenbar nicht gefolgt bzw. konnte ihm trotz des veränderten Tarifgefüges für den öffentlichen Dienst zumindest bis zum Jahr 2007 nicht folgen. Dies ist jedoch auch der langsamen Umstellung auf die neuen Tarife mit Übergangstarifen für die „alten“ Beschäftigten zuzuschreiben. Die prekäre Finanzlage vieler ostdeutscher Kommunen und einiger ostdeutscher Bundesländer führte zu Einsparungen, die sich an einem besonders starken Personalabbau ablesen lassen. Während sich die Beschäftigtenzahl des öffentlichen Dienstes im alten Bundesgebiet zwischen 1995 und 2007 um 12,2 \% reduzierte (von 4.337 .400 auf 3.807.400), betrug der prozentuale Rückgang in den ostdeutschen Bundesländern 20,1 \% (von 1.033.600 auf 733.200, Statistisches Bundesamt 2009). Auch der Anteil befristeter Beschäftigungsverhältnisse im öffentlichen Dienst war 2005 in den ostdeutschen Bundesländern mit 16,1 \% wesentlich höher als in den westdeutschen Bundesländern mit 12,4\% (Statistisches Bundesamt 2005, eigene Berechnung, ohne Auszubildende sowie Wehr-/Zivildienstleistende). Diese Befunde lassen vermuten, dass dort, wo die öffentlichen Arbeitgeber zu Sparmaßnahmen gezwungen und gleichzeitig an ein festes Tarifgefüge gebunden sind, eine Differenzierung zwischen einer geschützten Beschäftigtengruppe mit guten Einkom-

\footnotetext{
6 Für einige Quantile (speziell bei kleinem Sample) können der Standardfehler und die Konfidenzintervalle mit der Quantilsregression nach Koenker/Bassett (1978) nicht ermittelt werden. In diesen Fällen verweisen wir auf die Ergebnisse der ungewichteten Simultaneous Quantile Regression.
} 


\section{Abb. 2: Lohndifferenz zwischen öffentlichem Dienst und Privatwirtschaft nach Bildungsniveau 1995 und 2007 - in \% -}
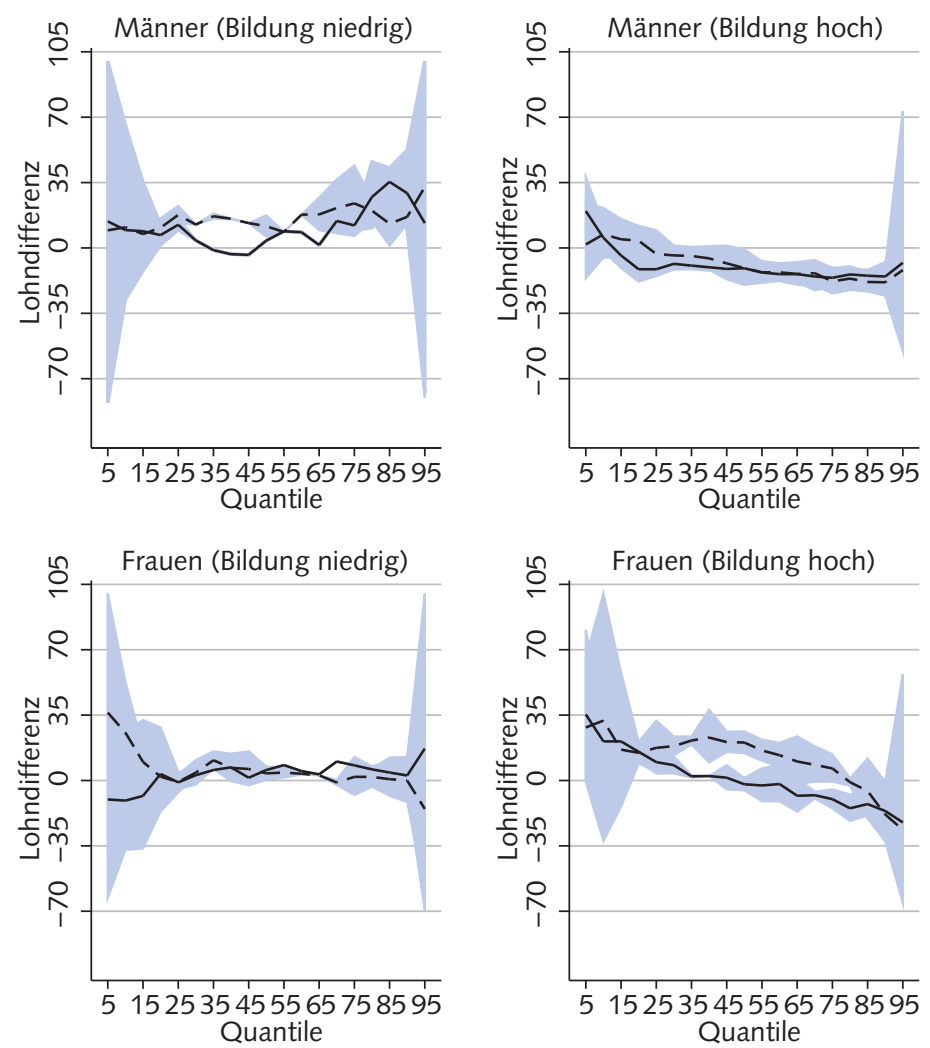

$--1995$

$-2007$

- 95 -\%-Konfidenzintervall (boostrapped mit 500 Wiederholungen)

Methode: Die in den vier Teilgrafiken dargestellten Schätzergebnisse basieren auf acht separaten Samples (jeweils 18-64-Jährige in Voll- oder Teilzeitbeschäftigung aus Westdeutschland). Die „Bildung niedrig“-Samples beinhalten alle Personen mit ISCED $=1$ bis 2. Die „Bildung hoch" ${ }^{-}$-Samples beinhalten alle Personen mit ISCED $=5$ bis 6 . Abhängige Variable: Bruttostundenlohn(In). Kontrollvariablen: Beamter, Familienstand, Alter, Bildung, Beschäftigungsdauer, Teilzeit, Beruf (Querschnittgewichte verwendet) Quelle: SOEP; Berechnungen der Autoren.

menschancen und einer weniger geschützten Beschäftigtengruppe mit dem Risiko des Arbeitsplatzverlustes stattfindet. ${ }^{7}$

\subsection{BILDUNGSEFFEKTE}

Spätestens seitdem die Kommunale Gemeinschaftsstelle für Verwaltungsvereinfachung das Neue Steuerungsmodell als deutsche Variante des New Public Management vorgelegt hat, haben betriebswirtschaftliche Prinzipien der Personalführung Einzug in die Verwaltungspraxis gehalten (Jann 2005; KGSt 1993). Mit den Reformen hat sich auch das Anforderungsprofil an die Beschäftigten der öffentlichen Verwaltung erweitert: Kaufmännisches Rechnungswesen, E-Government und Prozessoptimierung erfordern neue Fähigkeiten und Kenntnisse. Qualifiziertes Personal wird somit zu einer der wichtigsten Ressourcen staatlicher Leistungserbringung. Gleichzeitig wird befürchtet, dass es schwieriger wer- den wird, gerade die Hochqualifizierten, die in der Privatwirtschaft bessere Verdienstmöglichkeiten haben, für den öffentlichen Dienst zu rekrutieren. Bisher sind die Einkommenschancen verschiedener Qualifikationsgruppen im öffentlichen Dienst sehr eng mit dem Motiv des sozialen Ausgleichs zwischen den Bevölkerungsgruppen verknüpft. Sollten in Zukunft vermehrt Managementaufgaben anfallen, während einfache Aufgaben bzw. Spezialaufgaben ausgelagert werden, wird dies einem weiteren Akademisierungsbedarf der öffentlichen Verwaltung Vorschub leisten.

Es wurde wiederholt festgestellt, dass die Lohndifferenz zwischen öffentlicher und privater Beschäftigung mit steigendem Qualifikationsniveau zunimmt (Dustmann/Van Soest 1997; Dustmann/Van Soest 1998; Melly 2005; Ponthieux/Meurs 2005). In Abbildung 2 werden die prozentualen Lohndifferenzen zur Privatwirtschaft in Abhängigkeit vom Bildungsstand dargestellt. Der Bildungsstand wird dabei durch die International Standard Classification of Education (ISCED) gemessen. Im Folgenden beziehen sich unsere Ergebnisse ebenso wie im kommenden Abschnitt zu Tarifangestellten und Beamtenschaft nur auf Westdeutschland.

Abbildung 2 zeigt in erster Linie, dass bildungsspezifische Lohndifferenzen weniger im Verlauf als vielmehr in der Lage der geschätzten Lohndifferenzkurve zum Ausdruck kommen. Männer mit niedriger Bildung (bis Haupt-/Realschulabschluss ohne Berufsausbildung) profitieren demnach bei einer Beschäftigung im öffentlichen Dienst mit wenigen Ausnahmen von einer positiven Lohndifferenz zur Privatwirtschaft. Im öffentlichen Dienst beschäftigte Männer mit hoher Bildung (Fach-/Hochschulausbildung) hingegen sahen sich bereits ab dem 15. bzw. 25. Einkommensperzentil einer negativen Lohndifferenz gegenüber. Während sich Bildungsunterschiede im Männer-Sample und bei Frauen mit niedriger Bildung vorrangig in der $\mathrm{Ni}$ veauverschiebung der Lohndifferenzkurve äußern, reagiert die Lohndifferenzkurve für Frauen mit höherem Bildungsabschluss auch auf das Einkommensperzentil, d.h. wir beobachten denselben negativen Verlauf der Lohndifferenz wie in Abbildung 1. Bei hoch gebildeten Frauen ist die positive Lohndifferenz im unteren Einkommensbereich etwas höher als bei den Männern und wandelt sich erst bei höheren Einkommen als bei den Männern in eine negative Lohndifferenz. In diesem Zusammenhang hat sich zwischen 1995 und 2007 insofern eine tendenzielle Veränderung ergeben, als hoch gebildete Frauen 1995 erst ab dem 85. Einkommensperzentil im öffentlichen Dienst weniger verdienten als in der Privatwirtschaft, im Jahr 2007 allerdings bereits ab dem 40. Einkommensperzentil.

Insgesamt stimmen diese Ergebnisse mit dem in den vorangegangenen Abschnitten dargestellten Bild des Staates als Arbeitgeber überein: Er trägt zum sozialen

\footnotetext{
7 Im Gegensatz zur Privatwirtschaft sind befristete Stellen im öffentlichen Dienst jedoch in der Regel nicht mit Lohnabschlägen verbunden, weil für befristete und unbefristete Stellen dasselbe Tarifgefüge zum Einsatz kommt. Mit Umstellung auf TVÖD und TV-L haben sich eventuell für einen kleinen Teil der wiederholt befristet Beschäftigten, die mehrmals in die niedrigste Erfahrungsstufe eingestuft werden, Lohnnachteile ergeben. Dies kann durch den Vergleich von zwei Querschnittsdatensätzen des SOEP nicht überprüft werden.
} 
Ausgleich bei, indem er die Lohnspreizung zwischen den höchsten und den niedrigsten Einkommensgruppen und zwischen den Beschäftigten mit niedriger und mit hoher Bildung relativ gering hält. Für öffentlich Beschäftigte mit hoher Bildung bedeutet das Ergebnis unserer Analyse jedoch, dass sie im Vergleich zu Beschäftigten in der Privatwirtschaft tendenziell Nachteile erfahren. Angesichts der steigenden Konkurrenz um qualifizierte Beschäftigte wirft dies die Frage auf, ob geringere Bruttostundenlöhne im öffentlichen Dienst die Rekrutierung guten Personals verhindern. Bei dieser Frage muss jedoch auch berücksichtigt werden, dass sich die Qualität eines Arbeitgebers und seine Attraktivität nicht nur über die Entlohnungsfrage definieren. Die geringere Entlohnung von hoch qualifizierten Beschäftigten wird womöglich durch Arbeitsplatzsicherheit, Investitionen in die Aus- und Weiterbildung oder familienfreundliche Arbeitszeitmodelle ausgeglichen. Zudem bietet der öffentliche Dienst gesellschaftlich relevante Betätigungsfelder, die für Beschäftigte mit einer besonderen Motivation zur Erbringung öffentlicher Güter und Dienstleistungen attraktiv sind (Vandenabeele 2008). Ob sich die schlechteren Einkommenschancen für Hochqualifizierte negativ auf die Rekrutierungschancen des öffentlichen Dienstes auswirken oder nicht, wird demnach vermutlich auch davon abhängen, inwieweit die monetären durch nicht-montäre Lohnbestandteile kompensiert werden.

\subsection{BEAMTENSCHAFT UND TARIFBESCHÄFTIGTE}

Ein weiteres Beschäftigungsmerkmal betrifft die Unterscheidung zwischen Beamtinnen und Beamten einerseits und Tarifbeschäftigten andererseits. Das Festhalten an dieser Statusunterscheidung innerhalb des öffentlichen Dienstes ist politisch umstritten. Mit Blick auf die Einstellungspraxis ist ein Abbau der Unterschiede zwischen den Beschäftigungsformen kein vorrangiges verwaltungspolitisches Ziel. Der Beamtenstatus scheint vielmehr als Steuerungsinstrument genutzt zu werden, um die Personalkosten durch den Wegfall der arbeitgeberseitigen Sozialversicherungsbeiträge zumindest mittelfristig niedriger zu halten und um die Attraktivität schwer zu besetzender Stellen zu erhöhen. Bezüglich ihrer Arbeitsplatzsicherheit und ihrer sozialen Absicherung sind Beamtinnen

\section{Abb. 3: Brutto- und Nettolohndifferenzen zwischen öffentlichem Dienst und Privatwirtschaft, tarifangestellt vs. verbeamtet 1995 und 2007 - in \% -}
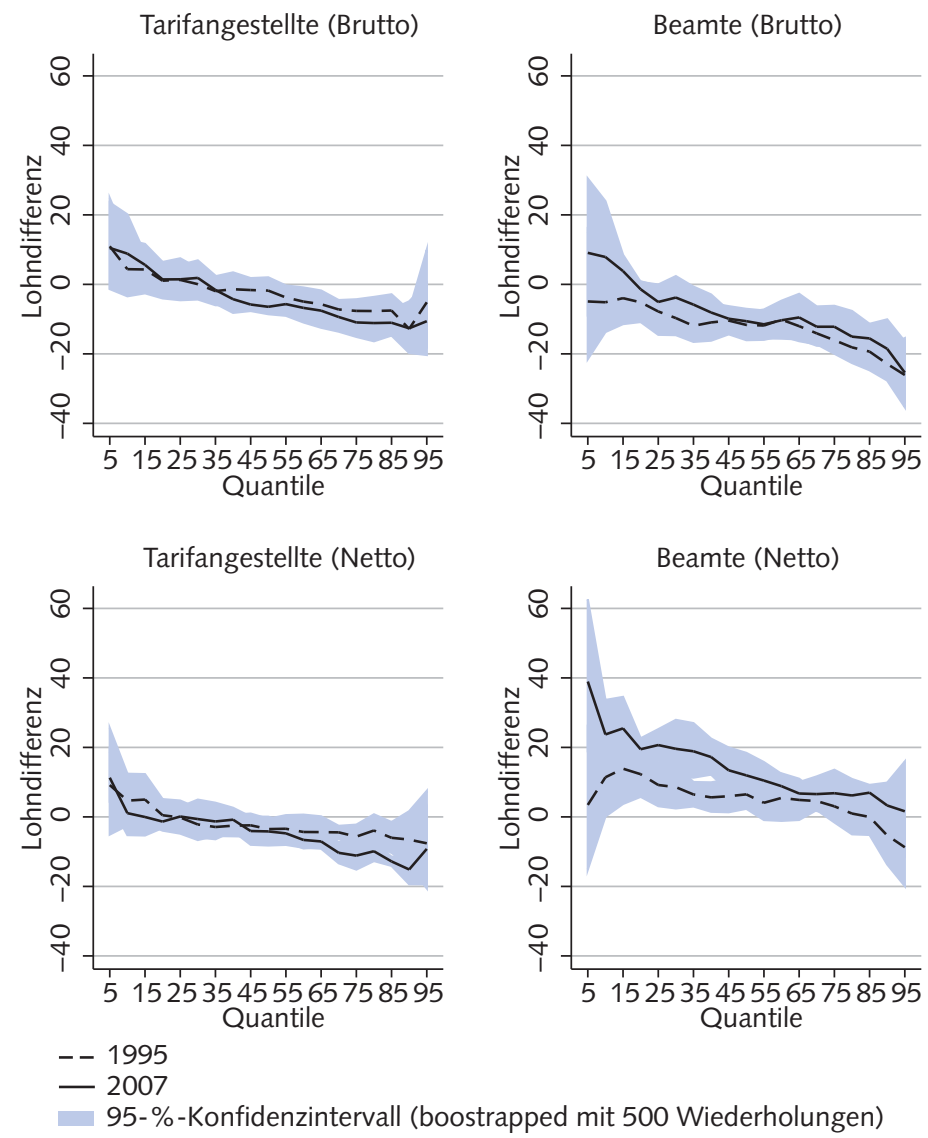

Methode: Die in den vier Teilgrafiken dargestellten Schätzergebnisse basieren auf vier separaten Samples (jeweils 18-64-jährige Männer aus Westdeutschland in Voll- oder Teilzeitbeschäftigung). Die Variablen Tarifangestellte und Beamte werden in einem Modell geschätzt. Abhängige Variable: Bruttostunden- bzw. Nettostundenlohn(In). Kontrollvariablen: Familienstand, Alter, Bildung, Beschäftigungsdauer, Teilzeit, Beruf (Querschnittgewichte verwendet) Quelle: SOEP; Berechnungen der Autoren. WSI MITTELLUNGEN

und Beamte gegenüber Tarifbeschäftigten im Vorteil. Es stellt sich jedoch die Frage, in welchem Umfang die Brutto- und Nettoerwerbslöhne zwischen diesen Beschäftigungskategorien im Vergleich zur Privatwirtschaft variieren. In Abbildung 3 wird im Gegensatz zu den vorherigen Abbildungen zwischen Brutto- und Nettolöhnen unterschieden, da sich die Nettolöhne der Beamtenschaft wegen fehlender Sozialversicherungsbeiträge weniger stark von ihren Bruttolöhnen unterscheiden, als dies bei Tarifbeschäftigten im öffentlichen Dienst der Fall ist.

Vergleicht man die Bruttostundenlöhne, so zeigt sich, dass Tarifbeschäftigte in den unteren Einkommensgruppen etwas höhere Bruttostundenlöhne erhalten als privatwirtschaftlich Beschäftigte und dass die Lohndifferenz erst ab dem 25. Einkommensperzentil negativ und mit steigendem Einkommen größer wird. Bei Beamtinnen und Beamten hingegen ist die Lohndifferenz nur für einen sehr kleinen Teil der Beschäftigten im untersten Einkommensbereich positiv, und dies auch erst im Jahr 2007. Die Kurve verläuft dann steiler als bei den Tarifbeschäftigten, d.h. in den mittleren und höheren Einkommensgruppen sehen sich diese Beschäftigten einer größeren prozentualen Lohndifferenz zu privatwirtschaftlich Beschäftigten gegenüber als Tarifbeschäftigte des öffentlichen Dienstes. Betrachtet man lediglich Bruttostundenlöhne, scheint die Beschäftigung beim Staat als Tarifbeschäftigter in den höheren Einkommensbereichen lukrativer zu sein als eine Beschäftigung im Beamtenverhältnis.

Der Blick auf die Lohndifferenzen von privatwirtschaftlich Beschäftigten und öffentlich Beschäftigten auf Basis der Nettoerwerbslöhne revidiert dieses Bild allerdings deutlich. Bei den Tarifbeschäftigen ist 
die Kurve im Vergleich zu den Bruttostundenlöhnen für das Jahr 1995 flacher; sowohl die positive Nettolohndifferenz zur Privatwirtschaft im unteren Einkommensbereich als auch die negativen Nettolohndifferenzen in den höheren Einkommensbereichen waren also geringer als die Bruttolohndifferenzen. Die Kurve für das Jahr 2007 ist im Bereich der mittleren und höheren Einkommen jedoch deutlich steiler, d.h. die negative Lohndifferenz zur Privatwirtschaft auf Grundlage der Nettolöhne war höher als auf Grundlage der Bruttolöhne. Bei den Beamtinnen und Beamten ergeben sich im Vergleich von Brutto- und Nettoerwerbslöhnen noch weitaus größere Differenzen. Während die Lohndifferenz zu privatwirtschaftlich Beschäftigten bei den Nettoerwerbslöhnen 1995 bereits bis zum 85. Einkommensperzentil im positiven Bereich lag, ist die Kurve für das Jahr 2007 sowohl steiler als auch nach oben verschoben. Im Vergleich zur Kurve für die Bruttostundenlöhne fällt die positive Lohndifferenz zwischen Beamtenschaft und Beschäftigten der Privatwirtschaft bei den Nettostundenlöhnen weitaus stärker aus. Im unteren Einkommensbereich hat der Staatsdienst 2007 von positiven Lohndifferenzen bis zu rund $40 \%$ profitiert. Dieses auffällige Auseinanderdriften der Nettoeinkommensdifferenzen zur Privatwirtschaft ist vermutlich auf die Veränderung der monatlichen Beiträge in der Sozialversicherung zurückzuführen. Im Durchschnitt sind diese seit 1995 kontinuierlich gestiegen, wovon Privatbeschäftigte und Tarifangestellte im öffentlichen Dienst gleichermaßen betroffen sind. Beamtinnen und Beamte profitieren jedoch besonders im unteren Einkommensbereich vom Wegfall der Sozialversicherungsbeiträge.

Im Gegensatz zur Bruttobetrachtung zeigt der Blick auf die Nettostundenlöhne, dass sich die Lohndifferenzen für Tarifangestellte und Verbeamtete im Vergleich zum Privatsektor über weite Teile der Einkommensverteilung systematisch unterscheiden. Dies deutet einerseits darauf hin, dass das klassische Entlohnungsmuster im Kern öffentlicher Beschäftigung, dem Beamtentum, noch am deutlichsten auftritt und der Vorteil, den Beamtinnen und Beamte durch den Wegfall von Sozialversicherungsbeiträgen haben, dabei implizit berücksichtigt wird. In diesem Bereich des öffentlichen Dienstes ist die staatliche Ausgleichsfunktion zwischen verschiedenen sozialen Gruppen offenbar am stabilsten.
Anderseits wird ebenso deutlich, dass bei der Betrachtung der Nettostundenlöhne der Grundsatz „Gleicher Lohn für gleiche Arbeit" im Falle der Tarifangestellten und der Beamtenschaft nicht erfüllt wird.

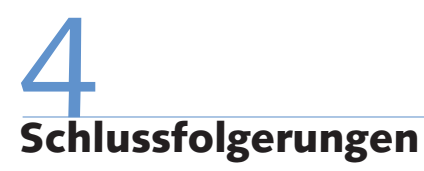

Bevor wir die Implikationen der empirischen Analyse thematisieren, sollen die Grenzen dieser Studie benannt werden. Trotz der Vorzüge, die die Quantilsregression bei der Untersuchung von Einkommensverteilungen bietet, sind die Ergebnisse ihrem Charakter nach deskriptiv. Zwischen den Jahren 1995 und 2007 können wenig systematische Unterschiede festgestellt werden (die Konfidenzintervalle der Lohndifferenzkurven überschneiden sich in der Regel deutlich). Dieser Befund deckt sich mit den Ergebnissen von Melly (2005). Für westdeutsche Männer, so stellen wir fest, ist die negative Lohndifferenz im oberen Einkommensbereich zwischen 1995 und 2007 tendenziell gestiegen. Ob diese Veränderung aus einem allgemeinen Anstieg der Spitzengehälter oder einem Absinken des Lohniveaus im öffentlichen Dienst resultiert, kann jedoch aus den Ergebnissen der Quantilsregression nicht abgelesen werden. Ein weiterer Aspekt, der nicht durch die Einführung von Kontrollvariablen behoben werden kann, betrifft die Tatsache, dass die Beschäftigten des öffentlichen Dienstes und der Privatwirtschaft insofern nicht direkt miteinander verglichen werden können, als sie sich hinsichtlich sozio-ökonomischer und berufsspezifischer Merkmale voneinander unterscheiden. Die Beschäftigten des öffentlichen Dienstes sind also in ihrer Zusammensetzung kein genaues Abbild der Erwerbsbevölkerung im Allgemeinen.

Doch auch wenn die Ergebnisse der Quantilsregression keine kausalen Abhängigkeiten abbilden, sind sie in der Lage, über staatliche Lohnstrukturen und die staatliche Lohnpolitik zu informieren. Unsere Untersuchung der Lohndifferenzen zwischen öffentlichem Dienst und Privatwirtschaft hat deutlich gemacht, dass unterschiedlichen Beschäftigtengruppen unterschiedliche Vor- und Nachteile aus einer Beschäftigung im öffentlichen Dienst erwachsen. Die zugespitzte Frage nach der Lukrativität des Staatsdienstes muss daher mit einem „das hängt davon ab “ beantwortet werden. Sie hängt vor allem davon ab, in welchem Abschnitt der Einkommensverteilung sich jemand befindet, doch auch von der regionalen Verortung des Arbeitsplatzes, vom Geschlecht und Bildungsniveau der Beschäftigten und von der Form des Beschäftigungsverhältnisses. So profitieren öffentlich Beschäftigte in Ostdeutschland in höherem Umfang von einer Beschäftigung im öffentlichen Dienst als ihre Kolleginnen und Kollegen in Westdeutschland. Und während Frauen bis in höhere Einkommensbereiche hinein (West) bzw. durchweg (Ost) im öffentlichen Dienst bessere Verdienstchancen haben als in der Privatwirtschaft, sind für westdeutsche Männer im öffentlichen Dienst fast durchweg beachtliche prozentuale Lohnnachteile zu konstatieren. In Bezug auf den Einfluss des Bildungsgrades auf die Lohndifferenz zwischen öffentlichem Dienst und Privatwirtschaft hat sich gezeigt, dass Personen mit niedriger Bildung mehr und Personen mit hoher Bildung weniger verdienen als im Privatsektor. Beamtinnen und Beamte profitieren unter Berücksichtigung der Nettostundenlöhne von deutlich ausgeprägteren Lohndifferenzen zur Privatwirtschaft als Tarifbeschäftigte im öffentlichen Dienst: Im unteren bis mittleren Einkommensbereich verdienen Erstere erheblich mehr als vergleichbare Beschäftigte der Privatwirtschaft, und ihr Lohnvorteil fällt wesentlich größer aus als bei den Tarifbeschäftigten.

Wie sind diese Ergebnisse vor dem Hintergrund des anhaltenden Personalabbaus im öffentlichen Dienst zu betrachten? Es kann vermutet werden, dass der Personalabbau im öffentlichen Dienst in engem Zusammenhang zu den vergleichsweise stabilen Entlohnungsstrukturen steht. Der Staat bietet weiterhin auch „unten“ privilegierte und gut bezahlte Beschäftigungsverhältnisse, hat jedoch gerade in diesem Beschäftigungssegment Personal abgebaut (Czerwick 2007). Obgleich diese Frage im Rahmen dieser Studie nicht abschließend beantwortet werden kann, legen die Reglungen im TVÖD und TV-L den Schluss nahe, dass sich diese Entwicklung tendenziell verstärkt hat. Insbesondere mit den untersten Tarifstufen wurde ein Niedriglohnbereich eingeführt, der den Niedriglohnbereich der Privatwirtschaft nachahmt. Dies ist aber bis zum Jahr 2007 offenbar noch nicht voll zum Tragen gekommen bzw. die Niedriglöhne in der Pri- 
vatwirtschaft sind nicht „eingeholt" worden. Im Sozialsektor gelingt es bereits seit einigen Jahren nicht mehr, dem Lohnniveau und den Beschäftigungsbedingungen des öffentlichen Dienstes zu folgen
(Kühnlein/Wohlfahrt 2006). Ob wir daher in naher Zukunft mit einer Zwei-KlassenGesellschaft im öffentlichen Sektor rechnen müssen, die sich nach Beamtenstatus, langjähriger Tarifbeschäftigung und Rand- gruppen in zeitlich begrenzten Beschäftigungsverhältnissen mit niedrigen Löhnen unterscheidet, müssen weitere Untersuchungen zur staatlichen Lohnpolitik zeigen.

\section{LITERATUR}

Ahlers, E. (2004): Beschäftigungskrise im öffentlichen Dienst? in: WSIMitteilungen 2, S. 78-83

Allington, N./Morgan, P. (2003): Does it Pay to Work in the Public Sector? Evidence from Three Decades of Econometric Analyses, in: Public Money \& Management 4, S. 253-262

Bull, H. P. (2006): Vom Staatsdiener zum öffentlichen Dienstleister: Zur Zukunft des Dienstrechts, Berlin

Czerwick, E. (2007): Die Ökonomisierung des öffentlichen Dienstes: Dienstrechtsreformen und Beschäftigungsstrukturen seit 1991, Wiesbaden

Disney, R./Gosling, A. (1998): Does It Pay to Work in the Public Sector?, in: Fiscal Studies 4, S. 347-374

Dustmann, C./Van Soest, A. (1997): Wage Structures in the Private and Public Sectors in West Germany, in: Fiscal Studies 3, S. 225-247

Dustmann, C./Van Soest, A. (1998): Public and Private Sector Wages of Male Workers in Germany, in: European Economic Review 42, S. 14171441

Hao, L./Naiman, D. (2007): Quantile Regression, London

Jaehrling, K. (2007): Wo das Sparen am leichtesten fällt. Reinigungs- und Pflegehilfskräfte im Krankenhaus, in: Bosch, G./Weinkopf, C. (Hrsg.): Arbeiten für wenig Geld. Niedriglohnbeschäftigung in Deutschland, Frankfurt a. M., S. 175-210

Jann, W. (2005): Neues Steuerungsmodell, in: Blanke, B./Bandemer, S./Nullmeier, F./Wewer, G.: (Hrsg.): Handbuch zur Verwaltungsreform, Wiesbaden, S. 74-83

Koenker, R./Bassett, G. (1978): Regression Quantiles, in: Econometrica 1 S. 33-50

Kommunale Gemeinschaftsstelle für Verwaltungsvereinfachung (KGSt) (1993): Neues Steuerungsmodell. Konturen, Begründung, Umsetzung, Kommunale Gemeinschaftsstelle für Verwaltungsvereinfachung, Köln
Kühnlein, G./Wohlfahrt, N. (2006): Soziale Träger auf Niedriglohnkurs? Zur aktuellen Entwicklung der Arbeits- und Beschäftigungsbedingungen im Sozialsektor, in: WSI-Mitteilungen 7, S. 389-395

Melly, B. (2005): Public-private Sector Wage Differentials in Germany: Evidence from Quantile Regression, in: Empirical Economics 30, S. 505520

Pollitt, C./Bouckaert, G. (2004): Public Management Reform: A Comparative Analysis, Oxford

Ponthieux, S./Meurs, D. (2005): The Gender Wage Gap in Europe: Women, Men and the Public Sector, Institut National de la Statistique et des Etudes Economiques, Paris

Reichard, C. (2005). Personalmanagement, in: Blanke, B./Bandemer, S./ Nullmeier, F./Wewer, G. (Hrsg.): Handbuch zur Verwaltungsreform, Wiesbaden, S. 229-234

Seidel, A./Ovey, J.-D./Birk, S. (2008): Zukunftsreport Moderner Staat 2008. Die öffentliche Verwaltung im Jahr 2020. Innovative Verwaltung, Special 3

Statistisches Bundesamt (2005): Mikrozensus, Wiesbaden

Statistisches Bundesamt (2009): Finanzen und Steuern 2008. Personal des öffentlichen Dienstes, Fachserie 14, Reihe 6, Erweiterung, Wiesbaden Tepe, M./Gottschall, K. /Kittel, B. (2008): Marktwirtschaftsmodelle und öffentliche Beschäftigungsregimes in der OECD, in: dms - der moderne staat 2, S. 377-398

Vandenabeele, W. (2008): Government Calling: Public Service Motivation as an Element in Selecting Government as Employer of Choice, in: Public Administration 4, S. 1089-1105 